\title{
Asistencias ante desastres para agricultores
}

Los huracanes Irma y María ocasionaron que muchos productores de Puerto Rico y las Islas Vírgenes sufrieran pérdidas de cultivos y ganado, y daños estructurales en sus operaciones. El Departamento de Agricultura de los Estados Unidos (USDA) se ha comprometido a aquellos productores elegibles a recibir asistencia para recuperarse ante estos desastres naturales.

IMPORTANTE: NRCS - USDA habilitó una línea directa para ofrecer asistencia ante desastres a los productores que han sido afectados por el huracán María: Asistencia ante desastres - Línea directa: (787) 303-0341

\section{¿Cuáles son los programas del USDA disponibles para asistencia luego de un desastre?}

EI USDA cuenta con varios servicios y programas de asistencia para agricultores afectados por un desastre. Los programas listados abajo son manejados por diferentes agencias del gobierno federal. Cada programa tiene diferentes requisitos de elegibilidad. Recomendamos que usted se comunique con la agencia que maneja el programa que le interese para verificar si es elegible para recibir ayudas. La información de contacto para su municipio se encuentra en el otro lado de la página.

\begin{tabular}{|c|c|c|c|c|}
\hline \multicolumn{5}{|c|}{ Programas de Asistencia ante Desastres } \\
\hline Programa / Incentivo & Tipo & Elegibilidad & Hoja Informativa & Agencia \\
\hline $\begin{array}{l}\text { Emergency Assistance for Livestock, } \\
\text { Honeybees, and Farm-Raised Fish } \\
\text { (ELAP) }\end{array}$ & Financiero & $\begin{array}{l}\text { Productores de ganado, abejas y peces que sufrieron pérdidas por } \\
\text { tiempos adversos y que no son cubiertos por otro programa de } \\
\text { desastre. }\end{array}$ & 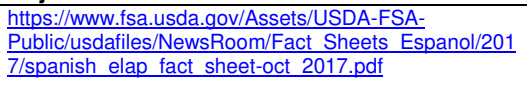 & $\begin{array}{l}\text { Agencia del Servicio } \\
\text { Agrícola (FSA) }\end{array}$ \\
\hline $\begin{array}{l}\text { Emergency Conservation Program } \\
\text { (ECP) }\end{array}$ & $\begin{array}{l}\text { Financiero y } \\
\text { Técnico }\end{array}$ & Productores con fincas que fueron dañadas por desastres naturales. & $\begin{array}{l}\text { https://www.fsa.usda.gov/Assets/USDA-FSA- } \\
\text { Public/usdafiles/NewsRoom/Fact Sheets Espanol/201 } \\
\text { 7lecp spanish factsheet sept2015.pdf }\end{array}$ & $\begin{array}{l}\text { Agencia del Servicio } \\
\text { Agrícola (FSA) }\end{array}$ \\
\hline Emergency Loan Program (ELP) & & $\begin{array}{l}\text { Productores con pérdidas de por } 10 \text { menos } 30 \% \text { debido a desastres } \\
\text { naturales. }\end{array}$ & $\begin{array}{l}\text { https://www.fsa.usda.gov/Assets/USDA-FSA- } \\
\text { Public/usdafiles/NewsRoom/Fact Sheets Espanol/201 } \\
\text { 7/spanish emergency loan program-oct 2017.pdf }\end{array}$ & $\begin{array}{l}\text { Agencia del Servicio } \\
\text { Agrícola (FSA) }\end{array}$ \\
\hline $\begin{array}{l}\text { Livestock Forage Disaster Program } \\
\text { (LFP) }\end{array}$ & Financiero & $\begin{array}{l}\text { Productores de ganado que han sufrido pérdidas de forraje debido a } \\
\text { sequia o fuegos. }\end{array}$ & $\begin{array}{l}\text { https://www.fsa.usda.gov/Assets/USDA-FSA- } \\
\text { Public/usdafiles/NewsRoom/Fact Sheets Espanol/201 } \\
5 / \text { /fp long fact sht } 2014 \text { spanish.pdf }\end{array}$ & $\begin{array}{l}\text { Agencia del Servicio } \\
\text { Agrícola (FSA) }\end{array}$ \\
\hline Livestock Indemnity Program (LIP) & Financiero & Productores con pérdida de ganado por tiempo adverso. & $\begin{array}{l}\text { https://www.fsa.usda.gov/Assets/USDA-FSA- } \\
\text { Public/usdafiles/NewsRoom/Fact Sheets Espanol/201 } \\
5 \text { 5/lip long fact sht } 2014 \text { spanish.pdf }\end{array}$ & $\begin{array}{l}\text { Agencia del Servicio } \\
\text { Agrícola (FSA) }\end{array}$ \\
\hline $\begin{array}{l}\text { Noninsured Crop Disaster Assistance } \\
\text { Program (NAP) }\end{array}$ & Financiero & $\begin{array}{l}\text { Productores con pérdida dado a desastres naturales y cuando el } \\
\text { seguro federal no está disponible. }\end{array}$ & $\begin{array}{l}\text { hntps://www.fsa.usda.gov/Assets/USDA-FSA- } \\
\text { Public/usdafiles/NewsRoom/Fact Sheets Espanol/201 } \\
\text { 7/spanish nap fact sheet-oct 2017.pdf }\end{array}$ & $\begin{array}{l}\text { Agencia del Servicio } \\
\text { Agrícola (FSA) }\end{array}$ \\
\hline Tree Assistance Program (TAP) & Financiero & $\begin{array}{l}\text { Productores de árboles y arbustos elegibles que fueron dañados por } \\
\text { desastres naturales. Arboles usados para pulpo o madera no son } \\
\text { elegibles. }\end{array}$ & $\begin{array}{l}\text { hitps://www.fsa.usda.gov/Assets/USDA-FSA- } \\
\text { Public/usdafiles/NewsRoom/Fact Sheets Espanol/201 } \\
\text { 7/spanish tap fact sheet-oct 2017.pdf }\end{array}$ & $\begin{array}{l}\text { Agencia del Servicio } \\
\text { Agrícola (FSA) }\end{array}$ \\
\hline Buy Up (Regular) Insurance & Seguro & $\begin{array}{l}\text { Productores asegurados por este seguro de la Corporación de } \\
\text { Seguros Agrícolas puede ser elegibles a recibir un } 65 \text { por ciento del } \\
\text { valor del mercado de la cosecha. }\end{array}$ & $\begin{array}{l}\text { hettps://www2.pr.gov/agencias/Agricultura/Documents/T } \\
\text { emas\%20de\%20interes/Porqu\%C3\%A9\%20es\%20ne } \\
\text { cesario\%20tener\%20una\%20p\%C3\%B3liza.pdf }\end{array}$ & $\begin{array}{l}\text { Corporación de } \\
\text { Seguros Agrícolas } \\
\text { (CSA) }\end{array}$ \\
\hline $\begin{array}{l}\text { Catastrophic Risk Protection } \\
\text { Endorsement (CAT) }\end{array}$ & Seguro & $\begin{array}{l}\text { Productores asegurados por la Corporación de Seguros Agrícolas con } \\
\text { pérdidas causadas por huracán u otro desastre natural. }\end{array}$ & 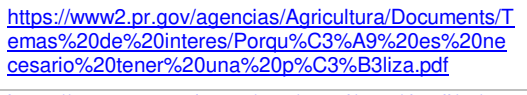 & $\begin{array}{l}\text { Corporación de } \\
\text { Seguros Agrícolas } \\
\text { (CSA) }\end{array}$ \\
\hline $\begin{array}{l}\text { Emergency Watershed Management } \\
\text { (EWM) }\end{array}$ & Cost share & $\begin{array}{l}\text { Dueños de terreno público o privado. Es necesario que sean } \\
\text { representados por un patrocinador. }\end{array}$ & $\begin{array}{l}\text { hitps://www.nrcs.usda.gov/wps/portal//nrcs/detail/pr/pro } \\
\text { grams/financial/ewp/?:cid=nrcs141p2 037246 }\end{array}$ & $\begin{array}{l}\text { Natural Resource } \\
\text { Conservation Service } \\
\text { (NRCS) }\end{array}$ \\
\hline
\end{tabular}

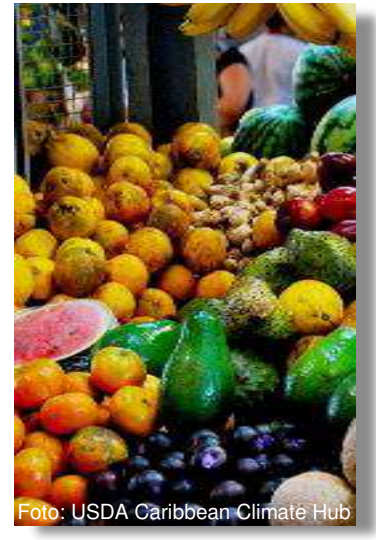




\section{Asistencias ante desastres para agricultores}

\section{¿Con cuál oficina me debo comunicar?}

La Agencia del Servicio Agrícola (FSA) y Servicio Nacional de Conservación de Recursos Naturales (NRCS) cuentan con oficinas en diferentes áreas de la isla que ofrecen asistencia a agricultores según su municipio de residencia. Busque su municipio en la tabla abajo para identificar con cuál oficina debe comunicarse.

\begin{tabular}{|c|c|c|c|c|}
\hline $\begin{array}{l}\text { Oficina Centro de } \\
\text { Servicio }\end{array}$ & Teléfono/Fax & Municipios Servidos & Dirección Física & Dirección Postal \\
\hline \multirow{2}{*}{\multicolumn{5}{|c|}{$\begin{array}{l}\text { Agencia del Servicio } \\
\text { Agrícola (FSA) } \\
\text { Puerto Rico }\end{array}$}} \\
\hline & & & & \\
\hline Arecibo & $\begin{array}{l}\mathrm{T}:(787) 878-2580 \\
\mathrm{~F}:(855) 415-2486\end{array}$ & $\begin{array}{l}\text { Arecibo, Barceloneta, Camuy, Ciales, Florida, Hatillo, Manatí, } \\
\text { Morovis, Quebradillas y Vega Baja }\end{array}$ & $\begin{array}{l}\text { FSA Service Center Office } \\
\text { Arecibo County Farm Service Agency } 1562 \\
\text { Miramar Ave., Suite } 202 \\
\text { Arecibo, PR 00612-2863 }\end{array}$ & $\begin{array}{l}\text { Caribbean Cinemas Building } 1562 \\
\text { Miramar Ave., STE } 202 \text { Arecibo, } \\
\text { PR 00612-2863 }\end{array}$ \\
\hline Barranquitas & $\begin{array}{l}\mathrm{T}:(787) 857-3940 \\
\mathrm{~F}:(855) 415-2487\end{array}$ & Aibonito, Barranquitas, Comerío y Orocovis & $\begin{array}{l}\text { FSA Service Center Office Barranquitas County } \\
\text { Farm Service Agency } \\
20 \text { Calle Padre Berrios STE } 1 \\
\text { Barranquitas, PR 00794-1640 }\end{array}$ & $\begin{array}{l}\text { USDA-FSA- Barranquitas Field } \\
\text { Office } \\
20 \text { Calle Padre Berrios STE } 1 \\
\text { Barranquitas, PR } 00794\end{array}$ \\
\hline Caguas & $\begin{array}{l}\text { T: (787) 743-2203 } \\
\text { F: (855) 415-2488 }\end{array}$ & $\begin{array}{l}\text { Aguas Buenas, Caguas, Canóvanas, Carolina, Cayey, Ceiba, Cidra, } \\
\text { Culebra, Fajardo, Guaynabo, Gurabo, Humacao, Juncos, Las } \\
\text { Piedras, Loíza, Luquillo, Naguabo, Río Grande, San Juan, San } \\
\text { Lorenzo, Trujillo Alto, Vieques y Yabucoa }\end{array}$ & $\begin{array}{l}\text { FSA Service Center Office } \\
\text { Caguas County Farm Service Agency Gatsby } \\
\text { Plaza, Floor \# 3, \#30 Calle Padial } \\
\text { Caguas, PR } 00725\end{array}$ & $\begin{array}{l}\text { Gatsby Plaza Bldg } \\
\text { Padial St. \#30, Suite } 310 \\
\text { Caguas, PR } 00725\end{array}$ \\
\hline Corozal & $\begin{array}{l}\text { T: (787) 859-3978 } \\
\text { F: (855) 415-2489 }\end{array}$ & $\begin{array}{l}\text { Bayamón, Cataño, Corozal, Dorado, Naranjito, Toa Alta, Toa Baja y } \\
\text { Vega Alta }\end{array}$ & $\begin{array}{l}\text { FSA Service Center Office } \\
\text { Corozal County Farm Service Agency } 29 \text { Calle } \\
\text { San Ramon STE } 1 \\
\text { Corozal, PR } 00783-2330\end{array}$ & $\begin{array}{l}29 \text { Calle San Ramon STE } 1 \\
\text { Corozal, PR 00783-2330 }\end{array}$ \\
\hline Lares & $\begin{array}{l}\mathrm{T}:(787) 897-4610 \\
\mathrm{~F}:(855) 415-2490\end{array}$ & Lares y San Sebastian & $\begin{array}{l}\text { FSA Service Center Office } \\
\text { Lares County Farm Service Agency State Road } \\
\# 129, \text { Km. 21.9, Callejones Ward } \\
\text { Lares, PR } 00669\end{array}$ & $\begin{array}{l}\text { USDA-FSA-Lares Field Office } \\
\text { Carretera } 129 \mathrm{Km} .21 .9 \\
\text { Bo. Callejones } \\
\text { Lares, PR } 00669\end{array}$ \\
\hline Ponce & $\begin{array}{l}\text { T: (787) 841-3136 } \\
\text { F: (855) 415-2492 }\end{array}$ & $\begin{array}{l}\text { Arroyo, Coamo, Guayama, } \\
\text { Guayanilla, Juana Díaz, Maunabo, Patillas, Peñuelas, Ponce, } \\
\text { Salinas, Santa Isabel, Villalba y Yauco }\end{array}$ & $\begin{array}{l}\text { FSA Service Center Office } \\
\text { Ponce County Farm Service Agency La Rambla } \\
\text { Tower } 606 \text { Tito Castro Ave., STE } 201 \\
\text { Ponce, PR } 00716\end{array}$ & $\begin{array}{l}\text { La Rambla Tower Suite } 201 \\
606 \text { Tito Castro Avenue } \\
\text { Ponce, PR } 00717\end{array}$ \\
\hline Mayagüez & $\begin{array}{l}\mathrm{T}:(787) 831-3328 \\
\mathrm{~F}:(855) 415-2491\end{array}$ & $\begin{array}{l}\text { Aguada, Aguadilla, Añasco, Cabo Rojo, Guánica, Hormigueros, } \\
\text { Isabela, Lajas, Las Marías, Maricao, Mayagüez, Moca, Rincón, } \\
\text { Sabana Grande y San German }\end{array}$ & $\begin{array}{l}\text { FSA Service Center Office } \\
\text { Mayagüez County Farm Service Agency } \\
637 \text { Sur Santa Teresa de Journet Ave., Bechara } \\
\text { BLDG } \\
\text { Mayagüez, PR } 00682\end{array}$ & $\begin{array}{l}\text { Bechara Building Suite } 2021 \\
637 \text { South Santa Teresa de } \\
\text { Journet Ave } \\
\text { Mayagüez, PR } 00682\end{array}$ \\
\hline Utuado & $\begin{array}{l}\mathrm{T}:(787) 894-1480 \\
\mathrm{~F}:(855) 415-2493\end{array}$ & Jayuya y Utuado & $\begin{array}{l}\text { FSA Service Center Office } \\
\text { Utuado County Farm Service Agency } \\
47 \text { F.L. Ribas Dominicci Ave. } \\
\text { Utuado, PR } 00641\end{array}$ & $\begin{array}{l}\text { USDA-FSA- Utuado Field Office } \\
47 \text { F.L. Ribas Dominicci Ave. } \\
\text { Utuado, PR } 00641\end{array}$ \\
\hline \multicolumn{5}{|c|}{ Gludu, $r$ novo4t } \\
\hline St. Croix & $\begin{array}{l}\text { T: }(340) 773-9146 \\
\text { ext } 100 \\
F:(855) 478-8386\end{array}$ & St. Croix, St. John y St. Thomas & $\begin{array}{l}\text { St. Croix County Farm Service Agency } \\
4401 \text { Estate Sion Farm Suite } 2 \text { Christiansted, VI } \\
00820\end{array}$ & $\begin{array}{l}\text { 4401 Sion Farm \#2 Christiansted, } \\
\text { VI 00820-4245 }\end{array}$ \\
\hline
\end{tabular}




\section{¿Tiene una finca? El USDA puede ayudarle.}

Pasos para solicitar a los programas de incentivos del USDA en el Caribe. Asistencia para agricultores, ganaderos y dueños de fincas privadas.

(1) ¿Su finca se encuentra en producción agrícola o ganadera?

Sí

Su finca se encuentra en producción agrícola o ganadera.

U. Únicamente le interesa la conservación de sus recursos naturales.

(2) ¿Qué desea hacer en su finca?

Ayuda en caso de

Ayuda para construcción

o desarrollos

Establecer una agrícolas o ganaderas

Desarrollo de mercados agrícolas o distribución

rurales

Conservación de toda o

una parte de su finca
Desarrollo de talleres o

proyectos de

conservación

(3) ¿Qué tipo de ayuda busca?
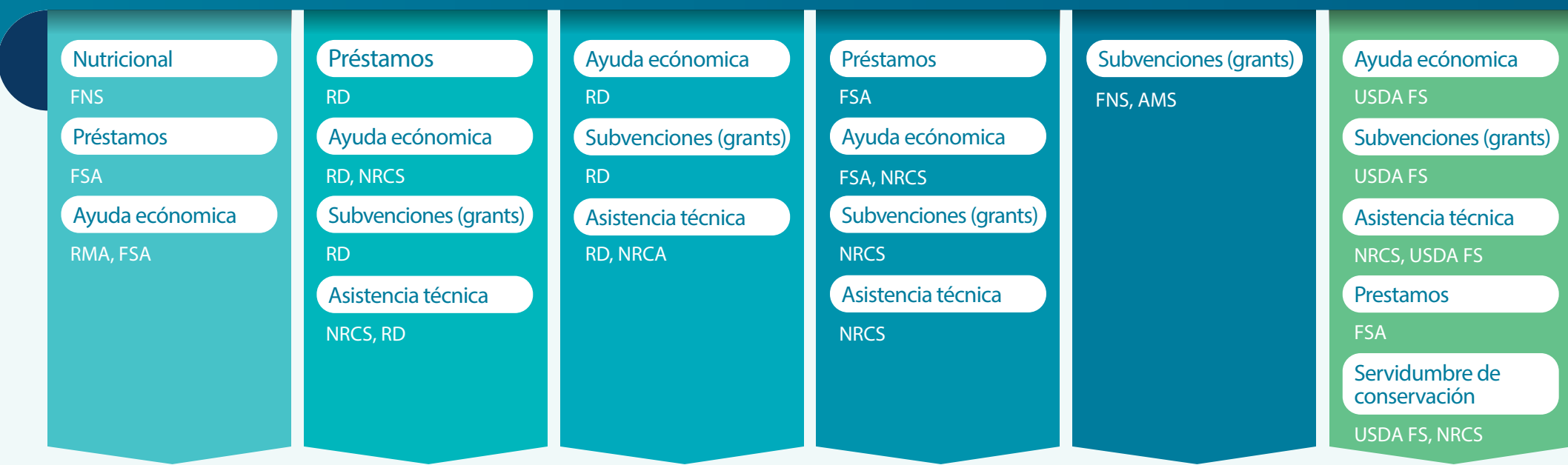

Ayuda ecónomica

USDA FS, NRCS

4 Agencias*1 del USDA

RMA*

Agencia ara la Administración

de Riesgos

(787) 829-2900 (PR)
RD

Desarrollo Rural

(787) 766-5096 (PR)

(340) 773-9146 (USVI)
FNS

Servicio de Alimentos y

Nutrición

(787) 766-5586 (PR)
AMS*3

Servicio de Mercadeo Agrícola
FSA

Agencia del Servicio

Agrícola

(787) 294-1616 (PR) (340) $773-9146$ (USVI)
NRCS

Servicio de Conservació

de Recursos Naturales

(787) 766-5206 (PR)

(340) 692-9632 (USVI)

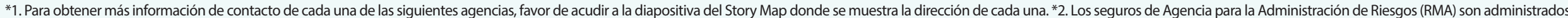

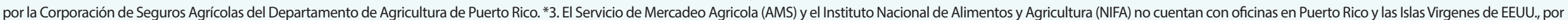

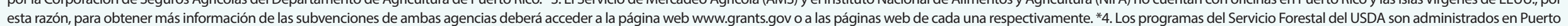
Rico por el Departamento de Recursos Naturales y Ambientales. En las Islas Virgenes de EEUU., algunos de estos programas son administrados por el Departamento de Agricultura local. 